PROCEEDINGS OF THE

AMERICAN MATHEMATICAL SOCIETY

Volume 132, Number 12, Pages 3725-3729

S 0002-9939(04)07371-X

Article electronically published on July 12, 2004

\title{
ON THE HOMOTOPY TYPE OF ESCHENBURG SPACES WITH POSITIVE SECTIONAL CURVATURE
}

\author{
L. ASTEY, E. MICHA, AND G. PASTOR \\ (Communicated by Jon G. Wolfson)
}

\begin{abstract}
A rigidity theorem is proved for principal Eschenburg spaces of positive sectional curvature. It is shown that for a very large class of such spaces the homotopy type determines the diffeomorphism type.
\end{abstract}

\section{INTRODUCTION}

The Eschenburg spaces introduced in 2, 3, 4, are quotients of free circle actions on $S U(3)$ and provide examples of non-homogeneous manifolds that admit a Riemannian metric with positive sectional curvature. In this work we shall be concerned with the family of principal Eschenburg spaces $E_{l, m}$ that fiber as circle bundles over the inhomogeneous Eschenburg flag manilfold $F^{\prime}$ of [4] and are obtained as quotient spaces of actions of the circle on $S U(3)$ given by

$$
z \cdot X=\operatorname{diag}\left(z^{l+m}, z^{l}, z^{m}\right) X \operatorname{diag}\left(z^{2 l+2 m}, 1,1\right)^{-1},
$$

where $l$ and $m$ are relatively prime integers and $\operatorname{diag}\left(z^{r}, z^{s}, z^{t}\right)$ denotes the diagonal matrix in $U(3)$ with entries $z^{r}, z^{s}$ and $z^{t}$. The topological and differentiable classification of this family was settled in [1] where it is shown that there are abundant examples of homeomorphic but not diffeomorphic principal Eschenburg spaces. Eschenburg also showed [4 that under the assumption that the product $l m$ is positive, the spaces $E_{l, m}$ admit a natural metric with positive sectional curvature. We shall refer to these spaces as principal positively curved Eschenburg spaces. Such spaces are very rigid, since it has been recently shown by Shankar [8] that homeomorphic principal positively curved Eschenburg spaces are in fact diffeomorphic. In this note we address the question of whether the homotopy type determines a unique principal positively curved Eschenburg space.

In Section 2, after characterizing the Eschenburg spaces $E_{l, m}$ as a set of integers in the quadratic field $\mathbb{Q}(\sqrt{5})$, we determine the orders of the fourth cohomology groups that can occur in this family. In Section 3 we first determine the precise number of principal positively curved Eschenburg spaces $E_{l, m}$ with prescribed fourth cohomology group. We then show that when the order of the fourth cohomology group satisfies a very general condition, the homotopy classification is enough

Received by the editors March 17, 2003 and, in revised form, June 12, 2003.

2000 Mathematics Subject Classification. Primary 53C20, 53C25, 57N65; Secondary 57R55.

Key words and phrases. Eschenburg spaces, homotopy types, diffeomorphism types.

Research supported by Conacyt grant 28783E and by Asociación Mexicana de Cultura, A.C.

(C)2004 American Mathematical Society 
to distinguish the diffeomorphism types of principal positively curved Eschenburg spaces.

\section{EsCHENBURG SPACES AS QUADRATIC INTEGERS}

It will be convenient to represent the Eschenburg spaces of type $E_{l, m}$ described above as integers in the quadratic field $\mathbb{Q}(\sqrt{5})$. We begin by recalling some wellknown facts about this field and refer the reader to [9] for details. The symbol $\mathbb{Q}(\sqrt{5})$ denotes the set of numbers $a+b \sqrt{5}$ where $a$ and $b$ are arbitrary rational numbers. If $\alpha=a+b \sqrt{5}$, then the conjugate of $\alpha$ is the number $\bar{\alpha}=a-b \sqrt{5}$ and the norm of $\alpha$ is given by $N(\alpha)=\alpha \bar{\alpha}=a^{2}-5 b^{2}$.

We will require as well some essentials about the arithmetic of $\mathbb{Q}(\sqrt{5})$. A quadratic integer is a number $\alpha=\frac{a}{2}+\frac{b}{2} \sqrt{5}$ in which $a$ and $b$ are (rational) integers with $a \equiv b \bmod 2$. Most of the basic properties of the divisibility of the integers generalize to the quadratic integers of $\mathbb{Q}(\sqrt{5})$, but one of the main differences lies in the fact that the group of units in $\mathbb{Q}(\sqrt{5})$ is infinite and, hence, every integer $\alpha$ in $\mathbb{Q}(\sqrt{5})$ has an infinite number of associates $\alpha \varepsilon$, with $\varepsilon$ a unit. However, it is not difficult to establish the following elementary result.

Lemma 2.1. Every quadratic integer $\alpha$ in $\mathbb{Q}(\sqrt{5})$ has a unique pair of associates $\alpha_{0}=\frac{a_{0}}{2}+\frac{b_{0}}{2} \sqrt{5}$ and $\alpha_{1}=\frac{a_{1}}{2}+\frac{b_{1}}{2} \sqrt{5}$ satisfying $a_{0}>0$ and $-a_{0}<5 b_{0}<a_{0}$, as well as $b_{1}>\left|a_{1}\right|$.

Definition 1. An integer $\alpha=\frac{a}{2}+\frac{b}{2} \sqrt{5}$ in $\mathbb{Q}(\sqrt{5})$ will be called basic if $a>0$ and $-a<5 b \leq a$.

Since the integers in $\mathbb{Q}(\sqrt{5})$ form a unique factorization domain, it follows from Lemma 2.1 that any quadratic integer $\alpha$ can be decomposed uniquely as a product $\alpha=\varepsilon \pi_{1} \pi_{2} \cdots \pi_{r}$, where $\varepsilon$ is a unit and the $\pi_{i}$ are basic primes. The next result, which is a direct consequence of Gauss's law of quadratic reciprocity, describes the basic primes in $\mathbb{Q}(\sqrt{5})$.

Proposition 2.2. Every basic prime in $\mathbb{Q}(\sqrt{5})$ is of one of the following types:

(i) $\alpha_{p}=p$, where $p$ is a rational prime with $p \equiv \pm 2 \bmod 5$.

(ii) $\alpha_{p}$ and $\bar{\alpha}_{p}$, where $N\left(\alpha_{p}\right)=N\left(\bar{\alpha}_{p}\right)=p$, for $p$ an odd rational prime with $p \equiv \pm 1 \bmod 5$.

(iii) $\alpha_{5}=\frac{5}{2}+\frac{1}{2} \sqrt{5}$.

The family of Eschenburg spaces $E_{l, m}$ is parametrized by pairs $(l, m)$ of relatively prime integers. The fourth cohomology group $H^{4}\left(E_{l, m} ; \mathbb{Z}\right)$ is cyclic of order $N$, where $N$ is the absolute value of $l^{2}+m^{2}+3 l m$. If we set $a=l-m$ and $b=l+m$, then there is a one-to-one correspondence between the Eschenburg spaces $E_{l, m}$ and the set of quadratic integers $\alpha=\frac{a}{2}+\frac{b}{2} \sqrt{5}$ in $\mathbb{Q}(\sqrt{5})$ with $\operatorname{gcd}(b+a, b-a)=2$.

Our first goal will be to determine which integers $N$ appear as orders of fourth cohomology groups of the family $E_{l, m}$. Observe first that since

$$
N(\alpha)=\frac{a^{2}}{4}-5 \frac{b^{2}}{4}=-\left(l^{2}+m^{2}+3 l m\right),
$$

the norm of $\alpha$ provides the order of the fourth cohomology group of the corresponding Eschenburg space. 
Let $\alpha=\frac{a}{2}+\frac{b}{2} \sqrt{5}$ be a quadratic integer with $\operatorname{gcd}(b+a, b-a)=2$ and let $p \neq 5$ be an odd rational prime such that $p \mid N(\alpha)$. If $p \equiv \pm 2 \bmod 5$, then $p \mid \frac{a}{2}+\frac{b}{2} \sqrt{5}$, implying that $p \mid a$ and $p \mid b$. This contradicts the assumption that $\operatorname{gcd}(b+a, b-a)=2$. Similarly, $N(\alpha)$ is odd, for otherwise $2 \mid \frac{a}{2}+\frac{b}{2} \sqrt{5}$, implying that 4 divides both $b+a$ and $b-a$. It follows that $\alpha=\frac{a}{2}+\frac{b}{2} \sqrt{5}$ is (up to a unit) a product of primes $\alpha_{p}$ and $\bar{\alpha}_{p}$, with $p \equiv \pm 1 \bmod 5$, and possibly $\alpha_{5}$. However, for each $p$ dividing $N(\alpha)$, only one of these two basic primes $\alpha_{p}$ and $\bar{\alpha}_{p}$ appears in the factorization of $\alpha$, for otherwise $p=\alpha_{p} \bar{\alpha}_{p}$ would divide $\frac{a}{2}+\frac{b}{2} \sqrt{5}$. Finally, the prime 5 turns out to be special in this respect, since $\alpha_{5}^{2}=5\left(\frac{3}{2}+\frac{1}{2} \sqrt{5}\right)$ cannot appear as a factor of $\alpha$. Thus we have the following result.

Proposition 2.3. Let $N$ be the order of the fourth cohomology group $H^{4}\left(E_{l, m} ; \mathbb{Z}\right)$. Then $N=5^{m} N^{\prime}$, where $m$ is 0 or 1 and $N^{\prime}$ is a product of powers of primes congruent to $\pm 1 \bmod 5$.

\section{Homotopy invariants of Eschenburg SPACES WITH POSITIVE SECTIONAL CURVATURE}

The geometric properties of the spaces $E_{l, m}$ were studied by Eschenburg [4], who established that these spaces admit a natural metric with positive sectional curvature provided that the product $l m$ is positive. It is immediate that a quadratic integer $\alpha=\frac{a}{2}+\frac{b}{2} \sqrt{5}$ represents a principal positively curved Eschenburg space if and only if $|b|>|a|$. We can now determine the precise number of principal positively curved Eschenburg spaces $E_{l, m}$ with a given fourth cohomology group.

Theorem 3.1. Let $N$ be as in Proposition 2.3, and let $k$ be the number of odd rational primes congruent with \pm 1 mod 5 that appear in the decomposition of $N$. Then there are exactly $2^{k+1}$ principal positively curved Eschenburg spaces $E_{l, m}$ such that $H^{4}\left(E_{l, m} ; \mathbb{Z}\right)$ is cyclic of order $N$.

Proof. We need to determine how many quadratic integers $\alpha=\frac{a}{2}+\frac{b}{2} \sqrt{5}$ satisfy $|N(\alpha)|=N$ with $\operatorname{gcd}(b+a, b-a)=2$ and $|b|>|a|$. The condition $\operatorname{gcd}(b+a, b-a)=2$ implies that at most one of $\alpha_{p}$ and $\bar{\alpha}_{p}$ can appear in the decomposition of $\alpha$ into primes. There are $2^{k}$ quadratic integers of the form $a_{5}^{m} \pi_{1}^{n_{1}} \pi_{2}^{n_{2}} \cdots \pi_{k}^{n_{k}}$, where each of the $\pi_{i}$ is either an $\alpha_{p}$ or an $\bar{\alpha}_{p}$. By Lemma 2.1 each of these quadratic integers has a unique associate $\frac{a_{j}}{2}+\frac{b_{j}}{2} \sqrt{5}$ satisfying $b_{j}>\left|a_{j}\right|$. The other $2^{k}$ integers are obtained by multiplying each $\frac{a_{j}}{2}+\frac{b_{j}}{2} \sqrt{5}$ by -1 .

We now address the question of finding two Eschenburg spaces $E_{l_{0}, m_{0}}$ and $E_{l_{1}, m_{1}}$ that admit metrics of positive sectional curvature with the same homotopy type. The homotopy classification of Eschenburg spaces was established by Milgram in [6]. (See also [5] for a partial classification.) For principal Eschenburg spaces, Milgram's result can be stated in terms of quadratic integers as follows. 
Theorem 3.2. Two quadratic integers $\alpha_{0}=\frac{a_{0}}{2}+\frac{b_{0}}{2} \sqrt{5}$ and $\alpha_{1}=\frac{a_{1}}{2}+\frac{b_{1}}{2} \sqrt{5}$ represent Eschenburg spaces with the same homotopy type if and only if

(i) $N\left(\alpha_{0}\right)=N\left(\alpha_{1}\right)=N$,

(ii) $b_{0} \equiv b_{1} \bmod 3$,

(iii) $b_{0}^{3} \equiv b_{1}^{3} \bmod N$ and

(iv) $\operatorname{gcd}\left(b_{i}+a_{i}, b_{i}-a_{i}\right)=2$.

Since we are only interested in Eschenburg spaces admitting a metric with positive sectional curvature and since $E_{l, m}$ is diffeomorphic to both $E_{m, l}$ and $E_{-l,-m}$, it is enough to consider pairs $\left(l_{0}, m_{0}\right)$ and $\left(l_{1}, m_{1}\right)$ with $l_{i}>m_{i}>0$. These inequalities are equivalent to

(v) $b_{i}>a_{i}>0$.

Let $\alpha_{0}=\frac{a_{0}}{2}+\frac{b_{0}}{2} \sqrt{5}$ and $\alpha_{1}=\frac{a_{1}}{2}+\frac{b_{1}}{2} \sqrt{5}$ be two quadratic integers satisfying conditions $(i)$ to $(v)$. The first and the last conditions imply that

$$
\left|b_{1}-b_{0}\right|<\sqrt{N}-\sqrt{\frac{4 N}{5}}=\frac{\sqrt{5}-2}{\sqrt{5}} \sqrt{N} .
$$

Let $p$ be a rational prime with $p^{n} \mid N$. By Proposition 2.3 we know that $p \geq 5$. Condition $(i v)$ implies that $\operatorname{gcd}\left(b_{0}, p\right)=\operatorname{gcd}\left(b_{1}, p\right)=1$. Hence, $\operatorname{gcd}\left(b_{0}^{3}, p^{n}\right)=1$. It is known (see [7, p. 104) that the congruence $x^{3} \equiv b_{0}^{3} \bmod p^{n}$ has either one or three solutions, according to whether $p \equiv 2$ or $p \equiv 1 \bmod 3$, respectively. Write $b_{1}=b_{0}+d$. Then $d \equiv 0 \bmod 3$; say $d=3 e$. Since $b_{0}^{3} \equiv b_{1}^{3} \bmod p^{n}$, if $p \equiv 2 \bmod 3$, then $b_{0} \equiv b_{1} \bmod p^{n}$, implying that $d \equiv 0 \bmod p^{n}$. Thus,

$$
e \equiv 0 \bmod p^{n} \text {. }
$$

We can now prove our main result.

Theorem 3.5. Suppose that $E_{l_{0}, m_{0}}$ and $E_{l_{1}, m_{1}}$ are principal positively curved Eschenburg spaces of the same homotopy type, and such that $l_{i}>m_{i}>0$. Let $N=$ $5^{m} p_{1}^{n_{1}} p_{2}^{n_{2}} \cdots p_{k}^{n_{k}}$, where $m$ is 0 or 1 and $p_{i} \equiv \pm 1 \bmod 5$, and write $N=x y$, where $x=5^{m} p_{1}^{n_{1}} \cdots p_{r}^{n_{r}}$ and $y=p_{r+1}^{n_{r+1}} \cdots p_{k}^{n_{k}}$ with $p_{i} \equiv 2 \bmod 3$ for $1 \leq i \leq r$ and $p_{i} \equiv 1$ $\bmod 3$ for $r+1 \leq i \leq k$. If $\frac{x}{y}>\frac{9-4 \sqrt{5}}{45}$, then $l_{0}=l_{1}$ and $m_{0}=m_{1}$.

Remark 3.6. Observe that since $\frac{9-4 \sqrt{5}}{45} \approx 0.00124$, the hypothesis $\frac{x}{y}>\frac{9-4 \sqrt{5}}{45}$ is not very restrictive.

Proof of 3.5. Write $b_{1}=b_{0}+3 e$ as above to obtain $\frac{\sqrt{5}-2}{3 \sqrt{5}} \sqrt{N}>e$ from (3.3). It follows from (3.4) that $x \mid e$. If we multiply by $x$ the inequality $x>\frac{9-4 \sqrt{5}}{45} y$, we get $x^{2}>\frac{9-4 \sqrt{5}}{45} N$. Taking square roots now gives $x>\frac{\sqrt{5}-2}{3 \sqrt{5}} \sqrt{N}>e$. But since $x \mid e$, then $e$ must be zero.

\section{REFERENCES}

1. L. Astey, E. Micha and G. Pastor, Homeomorphism and diffeomorphism types of Eschenburg spaces. Differential Geom. Appl. 7 (1997), 41-50. MR 98h:53072 
2. J. H. Eschenburg, New examples of manifolds with strictly positive curvature. Invent. Math. 66 (1982), 469-480. MR 83i:53061

3. J. H. Eschenburg, Cohomology of biquotients. Manuscripta Math. 75 (1992), 151-166. MR 93e:57070

4. J. H. Eschenburg, Inhomogeneous spaces of positive curvature. Differential Geom. Appl. 2 (1992), 123-132. MR 94j:53044

5. B. Kruggel, A homotopy classification of certain 7 manifolds. Trans. Amer. Math. Soc. 349 (1997), 2827-2843. MR 97m:55012

6. J. Milgram, The classification of Aloff-Wallach manifolds and their generalizations. Surveys on surgery theory, Vol 1. Annals of Math. Studies 145 (2000), 379-407. MR 2000m:57056]

7. I. Niven, H. Zuckerman and H. Montgomery, An introduction to the theory of numbers. 5th edition, John Wiley and Sons, New York, 1991. MR 91i:11001

8. K. Shankar, Strong inhomogeneity of Eschenburg spaces (with an Appendix by Mark Dickinson and Krishnan Shankar). Mich. Math. J. 50 (2002), 125-141. MR 2003g:53042

9. H. Stark, An introduction to number theory. MIT Press, Cambridge, Mass., 1978. MR 80a:10001

Departamento de Matemáticas, Centro de Investigación y Estudios Avanzados del IPN, Apartado Postal 14-740, México D.F. 07000

E-mail address: lastey@math.cinvestav.mx

Departamento de Matemáticas, Centro de Investigación y Estudios Avanzados del IPN, Apartado Postal 14-740, MÉXico D.F. 07000

E-mail address: emicha@math.cinvestav.mx

Instituto Tecnológico Autónomo de México (ItAm), Río Hondo No. 1, San Angel, MÉXICO D.F. 01000

E-mail address: pastor@itam.mx 